\title{
CCR2 mediates Helicobacter pylori-induced immune tolerance and contributes to mucosal homeostasis
}

\author{
Xia Sun $^{1,2} \mid$ Min Zhang ${ }^{2}$ | Mohamad El-Zaatari ${ }^{2}$ | Gray B. Huffnagle ${ }^{3}$ | John Y. Kao ${ }^{2}$
}

${ }^{1}$ Department of Pharmacology, School of Medicine, Shandong University, Jinan, Shandong, China

${ }^{2}$ Department of Internal Medicine, Division of Gastroenterology, University of Michigan Health System, Ann Arbor, MI, USA

${ }^{3}$ Department of Internal Medicine, Division of Pulmonary and Critical Care

Medicine, University of Michigan Health

System, Ann Arbor, MI, USA

\section{Correspondence}

John Y. Kao, Department of Internal Medicine, Division of Gastroenterology, University of Michigan Health System, Ann Arbor, MI, USA. Email: jykao@med.umich.edu

Xia Sun, Department of Pharmacology, School of Medicine, Shandong University, Jinan,

Shandong, China.

Email: sunxia@sdu.edu.cn

Funding information

Postdoctoral Innovation Project of Shandong Province, Grant/Award Number: 201402029; National Institute of Diabetes and Digestive and Kidney Diseases, Grant/Award Number: R01 DK087708-01; China Postdoctoral Science Foundation, Grant/Award Number: 2014M551917; National Natural Science Foundation of China, Grant/Award Number: 81302770

\begin{abstract}
Background: We previously demonstrated that $H$. pylori infection leads to increased induction of regulatory $T$ cells in local and systemic immune compartments. Here, we investigate the role of CCR2 in the tolerogenic programing of dendritic cells in a mouse model of $H$. pylori infection.

Materials and Methods: CCR2 deficient (CCR2KO) mice and wild-type (Wt) mice infected with $H$. pylori SS1 strain were analyzed by qPCR and FACS analysis. In vitro, bone marrow-derived DC on day 6 from CCR2KO and Wt mice cocultured with or without $H$. pylori were examined to determine the impact of CCR2 signaling on dendritic cells function by qPCR, ELISA, and FACS analyses.

Results: Acute $H$. pylori infection was associated with a threefold increase in CCR2 mRNA expression in the gastric mucosa. H. pylori-infected CCR2KO mice exhibited a higher degree of mucosal inflammation, that is, increased gastritis scores and proinflammatory cytokine mRNA levels, but lower degree of $\mathrm{H}$. pylori gastric colonization compared to infected Wt mice. Peripheral $H$. pylori-specific immune response measured in the CCR2KO spleen was characterized by a higher Th17 response and a lower Treg response. In vitro, CCR2KO bone marrow-derived DC was less mature and shown a lower Treg/Th17 ratio. Moreover, blockade of CCR2 signaling by MCP-1 neutralizing antibody inhibited $\mathrm{H}$. pylori-stimulated bone marrow-derived DC maturation.

Conclusions: Our results indicate that CCR2 plays an essential role in $\mathrm{H}$. pylori-induced immune tolerance and shed light on a novel mechanism of CCR2-dependent DC Treg induction, which appears to be important in maintaining mucosal homeostasis during H. pylori infection.
\end{abstract}

KEYWORDS

adaptive immunity, Chemokine receptor 2 , dendritic cells, immature, immune tolerance

\section{1 | INTRODUCTION}

Helicobacter pylori (H. pylori) colonizes the human stomach and contributes to diseases such as gastric ulcers and cancers. It is a highly adaptive Gram-negative bacterium, capable of evading immune surveillance despite systemic and mucosal humoral immunity. ${ }^{1,2}$ There is growing evidence that the failure of the host to eradicate $H$. pylori may be due to the ability of $H$. pylori to induce a regulatory T-cell (Treg) response against helper T-cell immunity. H. pylori-specific Tregs were recently shown to suppress memory T-cell responses to $\mathrm{H}$. pylori infection in individuals. ${ }^{3,4}$ In patients, the mRNA expression of Foxp3, a specific Treg surface marker, is higher in the gastric tissue of $\mathrm{H}$. pylori-infected persons compared with uninfected controls. ${ }^{5-7}$ Also, Harris et al. reported an inverse correlation between gastric 
Foxp3 expression and gastric pathology in $\mathrm{H}$ pylori-infected children compared to adults. ${ }^{8}$ Our laboratory has also shown that $H$. pylori can induce Treg development by DC that lead to the inhibition of the host immune response against $H$. pylori. ${ }^{9,10}$

The maturation and activation state of DC are regarded as a control point for the induction of either peripheral tolerance or autoimmunity. DC maturation has been shown to be associated with changes in the expression of chemokine ( $\mathrm{C}-\mathrm{C}$ motif) receptor 2 (CCR2), the receptor for monocyte chemoattractant protein- $1,{ }^{11}$ which is crucial for mucosal recruitment during inflammation. ${ }^{12-16}$ CCR2 has been shown to contribute to leukocyte trafficking and the control of intracellular pathogens as CCR2 knockout (CCR2KO) mice were unable to clear infection by Listeria monocytogenes. ${ }^{17}$ Deficiency of CCR2 was shown to impair DC trafficking to draining lymph nodes (LNs) in mice and resulting in a defective Th1 response and an increased susceptibility to L. major. $^{18}$ Thus, we speculate that CCR2 signaling may be critical in DC-mediated H. pylori tolerogenic response.

In this study, we examined the role of CCR2 in the recruitment and function of DC during $H$. pylori infection. In vivo analyses following acute $H$. pylori infection revealed no significant difference in gastric mucosal DC between wild-type $(\mathrm{Wt})$ and CCR2KO mice, suggesting CCR2 is not required for DC recruitment. However, chronic H. pylori infection showed more severe gastritis and a lower degree of gastric $\mathrm{H}$. pylori colonization in CCR2KO mice compared to their Wt mice. Splenic H. pylori antigen-specific helper T-cell cytokine analyses revealed a lower Treg/Th17 response ratio in chronically infected CCR2KO mice compared to Wt littermates. In vitro, CCR2KO bone marrow-derived DC (BMDC) were less mature and had defective T-cell priming function characterized by a lower Treg/Th17 response ratio, similar to that observed in in vivo. Neutralization of MCP-1, a CCR2 ligand, resulted in decreased DC maturation. Thus, our study demonstrates that CCR2 signaling is critical for induction of $H$. pylori-specific Treg response and may represent a major target in the modulation of the host response to $H$. pylori.

\section{2 | MATERIALS AND METHODS}

\section{$2.1 \mid$ Mice}

Male Wt C57BL/6 mice were purchased from Charles River and C57BL/6 CCR2KO mice were provided by Dr. Bethany Moore (Department of Internal Medicine, Division of Pulmonary and Critical Care Medicine, University of Michigan Health System, Ann Arbor, MI). Mice used were 8-10 weeks old. All animals were housed in the animal maintenance facility at the University of Michigan Health System. This research was undertaken with the approval of the University Committee on Use and Care of Animals at the University of Michigan (PRO00005890).

\subsection{Bacterial strains and culture condition}

H. pylori SS1 strain was cultured on Campylobacter-selective agar (BD Diagnostics, Bedford, MA, USA) for 3 days in a humidified microaerophilic chamber at $37^{\circ} \mathrm{C}$ (BBL Gas System, with CampyPak Plus packs, BD Biosciences San Jose, CA) as previously described. ${ }^{9}$ H. pylori infection of mice was achieved by oral gavage of $10^{9}$ bacteria suspended in $100 \mu \mathrm{L}$ of Brucella broth.

\subsection{Generation and stimulation of bone marrow- derived dendritic cells (BMDCs)}

BMDC from Wt or CCR2KO mice were derived using mouse GM-CSF $(10 \mathrm{ng} / \mathrm{mL})$ and IL-4 $(10 \mathrm{ng} / \mathrm{mL})$ and cultured with RPMI containing $10 \%$ fetal bovine serum (FBS) as previously described. ${ }^{19}$ BMDC were harvested and enriched $\left(10^{6}\right.$ cells $/ \mathrm{mL}$ ) by gradient centrifugation using OptiPrep density solution (Sigma, St. Louis, MO) according to manufacturer's instruction. For $H$. pylori-stimulated BMDC experiments, BMDC were stimulated with $10^{7} \mathrm{CFU} / \mathrm{mL}$ of $\mathrm{H}$. pylori or PBS for 18 hours and then cocultured with syngeneic splenocytes for 72 hours in a mixed leukocyte reaction (splenocyte: BMDC ratio of 10:1) as previously described. ${ }^{19}$

\subsection{Animal studies}

For acute infection, Wt and CCR2KO mice were infected for 24 hours with $10^{9} \mathrm{CFU} / \mathrm{mL}$ H. pylori SS1 via oral gavage. For chronic infection, mice were gavaged with $H$. pylori SS1 (three times over 1 week) and analyzed after 2 months. Six to ten animals per group (Wt or CCR2KO group) were used in each experiment. The stomachs from Wt and CCR2KO mice were cut along the greater curvature, and 2-mm strips of fundus and antrum were embedded in Tissue-Tek optimum cutting temperature compound (Sakura Finetek, Torrance, CA). Splenocytes from Wt and CCR2KO mice were cocultured for 72 hours with BMDC from Wt mouse. The splenocyte-to-BMDC ratio was 10-1. After 72 hours, splenocytes were collected, and the percentages and mRNA expression of IFN- $\gamma$, IL-17A, and Foxp3 were measured by FACS and qPCR, respectively.

\section{5 | Gastritis score determination}

After two-month infection with $\mathrm{H}$. pylori, the mice were euthanized. The stomachs of $\mathrm{Wt}$ and CCR2-deficient mice were removed and prepared for later analysis. Two adjacent full-thickness longitudinal strips were removed from the greater curvature of the stomach and fixed in formalin for histologic analysis (100 × magnification). The specimens were scored separately for the presence or absence of gastritis. The results were reported as the percentage of the number of gastritiscontaining fields per total number of fields examined on each histologic slide. ${ }^{20}$

\section{6 | qRT-PCR}

Total RNA from stomach, DC, or splenocytes was prepared using the RNeasy Mini Kit (QIAGEN, Hilden, Germany). Samples were reversetranscribed using iScript ${ }^{\mathrm{TM}}$ cDNA Synthesis Kit (BIO-RAD, Hercules, California). Expression of CCR2, H. pylori 16S, IL-6, IL-1 $\beta$, IL-12p35, 
TABLE 1 Primers used for the amplification of each gene

\begin{tabular}{|c|c|}
\hline Gene & Primer $\left(5^{\prime}-3^{\prime}\right)$ \\
\hline GAPDH & $\begin{array}{l}\text { F:5'-TCAAGAAGGTGGGTGAAGCAGG-3' } \\
\text { R:5'-TATTATGGGGGTCTGGGATGG-3' }\end{array}$ \\
\hline CCR2 & $\begin{array}{l}\text { F: 5'-GGAGAA AAGCCA ACT CCT TC-3' } \\
\text { R: 5'-AGG CAG TTGCAA AGG TACTG-3' }\end{array}$ \\
\hline IL-6 & $\begin{array}{l}\text { F:5'-CTACCCCAATTTCCAATGCT-3' } \\
\text { R:5'-ACCACAGTGAGGAATGTCCA-3' }\end{array}$ \\
\hline IL-17A & $\begin{array}{l}\text { F:5'-GCTCCAGAAGGCCCTCAGA-3' } \\
\text { R:5'-AGCTTTCCCTCCGCATTGA-3' }\end{array}$ \\
\hline IL-1 $\beta$ & $\begin{array}{l}\text { F:5'-CCAACTGGTACA TCAGCAC-3' } \\
\text { R:5'-TCTGCTCATTCACGAAAA GG-3' }\end{array}$ \\
\hline TNF- $\alpha$ & $\begin{array}{l}\text { F:5'-CCCACTCTGACCCCTT TACT-3' } \\
\text { R:5'-TTTGAGTCCTTGATGGTG GT-3' }\end{array}$ \\
\hline IL-10 & $\begin{array}{l}\text { F:5'-AGTGGAGCAGGTGAAGAGTG-3' } \\
\text { R:5'-TTCGGAGAGAGGTACAAACG-3' }\end{array}$ \\
\hline IL-12p35 & $\begin{array}{l}\text { F: 5'-ACGGCCAGGAAAAACTGAA-3' } \\
\text { R:5'-CTACCAAGGCACAGGGTGCAT-3' }\end{array}$ \\
\hline $\mathrm{IFN}-\gamma$ & $\begin{array}{l}\text { F:5'-TCAAGTGGCATAGATGTGGAAGAA-3' } \\
\text { R:5'-TGGCTCTGCAGGATTTTCATG-3' }\end{array}$ \\
\hline Foxp3 & $\begin{array}{l}\text { F:5'-TCTCCAGGTTGCTCAAAGTC-3' } \\
\text { R:5'-GCAGAAGTTGCTGCTTTAGG-3' }\end{array}$ \\
\hline HP $16 \mathrm{~s}$ & $\begin{array}{l}\text { F:5'-CAAGTCATGGCCCTTAC-3' } \\
\text { R:5'-TTGCGATTACTAGCGATTCC-3' }\end{array}$ \\
\hline
\end{tabular}

IL-10, IFN- $\gamma$, IL-17A, Foxp3, and GAPDH was measured using $\mathrm{iQ}^{\mathrm{TM}}$ SYBR Green Supermix Kit obtained from BIO-RAD. Primers are shown in Table 1. Finally, quantitation of relative differences in expression was calculated using the comparative $2^{-\Delta \Delta C T}$ method. ${ }^{21}$

\section{7 | Generation of single-cell suspensions and flow cytometry}

Stomachs and spleens from Wt and CCR2KO mice were digested using collagenase as described. ${ }^{22}$ Single-cell suspensions were preincubated with FcBlock (2.4G2) (BD Biosciences) to minimize nonspecific protein binding. Cells were stained with fluorochrome-conjugated Abs against surface markers MHCII, CD45, CD11b, CD11c, CD103, CCR2, CD80, and CD86 (eBioscience, San Diego, CA, USA) in staining buffer (PBS containing 2\% FCS and $0.1 \% \mathrm{NaN}_{3}$ ) for 30 minutes at room temperature and then washed. For staining of CD4, Foxp3, IFN- $\gamma$, and IL-17A, cells were permeabilized and subsequently stained. Samples were measured on a Coulter XL Flow Cytometer (Beckman Coulter, Miami, FL) and analyzed with FlowJo software (C6).

\section{8 | Statistical analyses}

Experiments were performed in triplicate, and all data were expressed as mean \pm SEM. Continuous ratio scale data were evaluated by unpaired Student $t$ test (for comparison between two samples) or by ANOVA (for multiple comparisons) or Turkey post hoc analysis, which compares treatment groups to a specific control group by GraphPad Prism software version 5.0c (GraphPad Software, La Jolla, CA, USA).
Significant values were indicated as follows: ${ }^{*} P<.05,{ }^{* *} P<.01$, and ${ }^{* * *} P<.001$.

\section{3 | RESULTS}

\section{1 | Acute H. pylori infection induced upregulation of CCR2 expression in mouse stomach but deficiency in CCR2 did not prevent DC accumulation in H. pylori-infected stomach}

To determine whether CCR2 is significantly upregulated after H. pylori infection, we measured gastric mucosal mRNA level of CCR2 24 hours after $H$. pylori infection (Figure $1 \mathrm{~A}$ ). A significant increase in CCR2 expression was found in the stomach 24 hours after $H$. pylori infection (Figure 1B). To examine the role of CCR2 on DC recruitment, we measured gastric mucosal $\mathrm{CD} 11 \mathrm{c}^{+} \mathrm{CD} 103^{+} \mathrm{DC}$ and $\mathrm{CD} 11 \mathrm{c}^{+} \mathrm{CD} 11 \mathrm{~b}^{+}$ macrophages during $H$. pylori infection. Acute $H$. pylori infection increased the percentage of DC but not macrophages in the stomach of $\mathrm{H}$. pylori-infected mice (Figure 1D). In CCR2KO mice (the lack of CCR2 was verified as shown in the right panels of Figure $1 \mathrm{C}$ ), there was no significant difference in the percentage of DC after $\mathrm{H}$. pylori infection between infected Wt and CCR2KO mice. These data indicate that CCR2 deficiency does not significantly alter DC recruitment to the gastric tissue and may play another role during H. pylori infection.

\subsection{CCR2 deficiency increased H. pylori-induced gastritis and decreased $H$. pylori colonization}

To further explore the role of CCR2 signaling during $H$. pylori infection, Wt and CCR2KO mice were chronically infected for two months with H. pylori (Figure 2A). We measured a higher gastritis score (Figure 2B,C) and increased expression of proinflammatory cytokines (eg, TNF- $\alpha$, IL-1 $\beta$, IL-12p35, IFN- $\gamma$, IL-17A, IL-6) in H. pylori-infected CCR2KO mice compared to infected $\mathrm{Wt}$ mice (Figure 2D). Next, we examined whether CCR2 signaling during chronic H. pylori infection impacts bacterial colonization using quantitative PCR. After two-month infection, we found a lower gastric level of $\mathrm{H}$. pylori colonization in CCR2KO mice compared to $\mathrm{Wt}$ mice (Figure $2 \mathrm{E}$ ). These data indicate that CCR2 signaling contributes to $\mathrm{H}$. pylori colonization in mouse stomach.

\section{3 | CCR2 deficiency skews H. pylori-specific Treg/ Th17 response toward Th17 Immunity}

To further study the mechanisms of how CCR2 deficiency leads to increased H. pylori gastritis, we examined the systemic H. pylori antigenspecific $\mathrm{T}$-cell responses by ex vivo stimulation of splenic $\mathrm{T}$ cells with $H$. pylori antigen and measured T-cell cytokine expression profiles (Figure 3A). In CCR2KO mice, H. pylori infection resulted in a Th17 skewed response with lower Treg and Th1 responses (Figure 3B), thus lowering the Treg/Th17 balance resulting in enhanced $H$. pylori-induced inflammation (Figure $3 \mathrm{C}$ ). This finding suggests that CCR2 regulates the balance of H. pylori-specific Treg priming and thus contributes to H. pylori tolerance, which in part explains the increased gastritis observed in CCR2KO mice. 
(A)

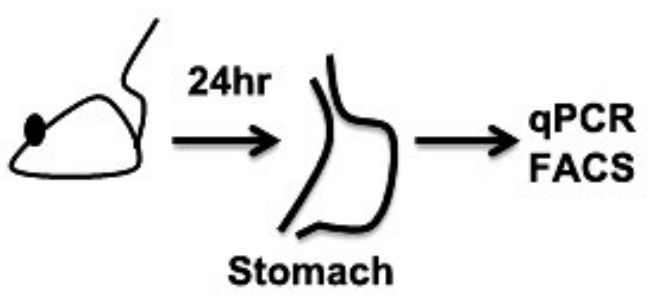

(B)

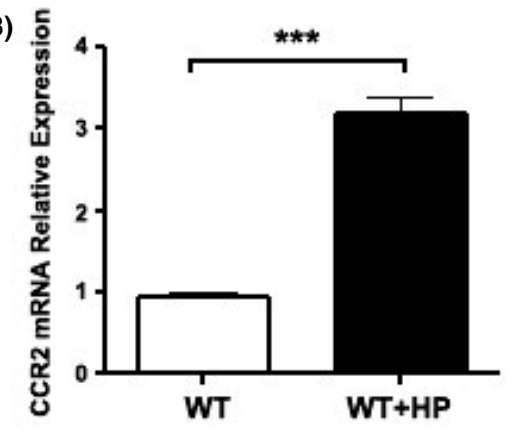

(C)

Uninfected
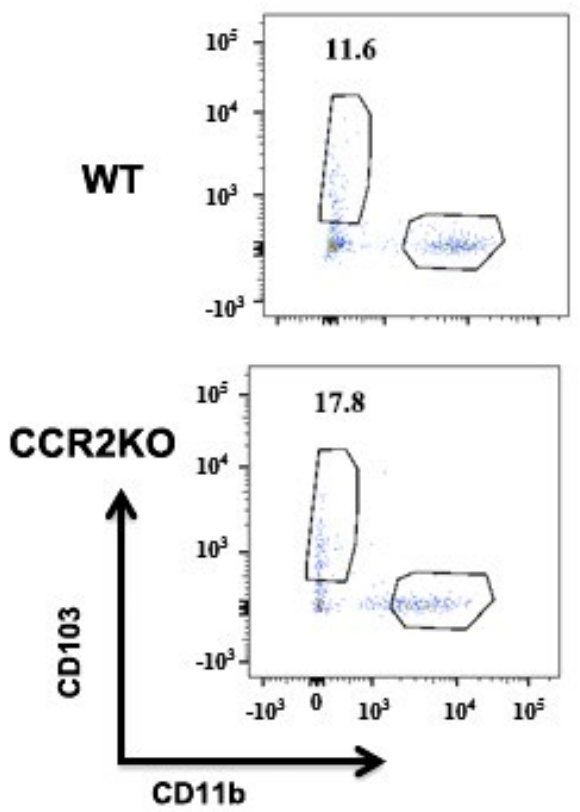

(D)

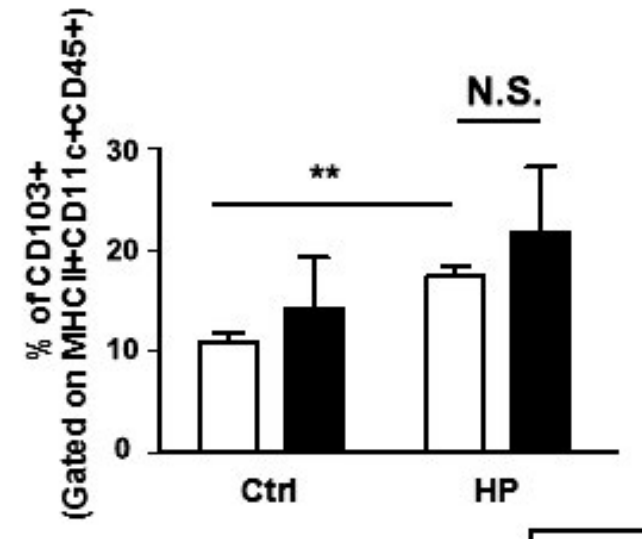

H. pylori
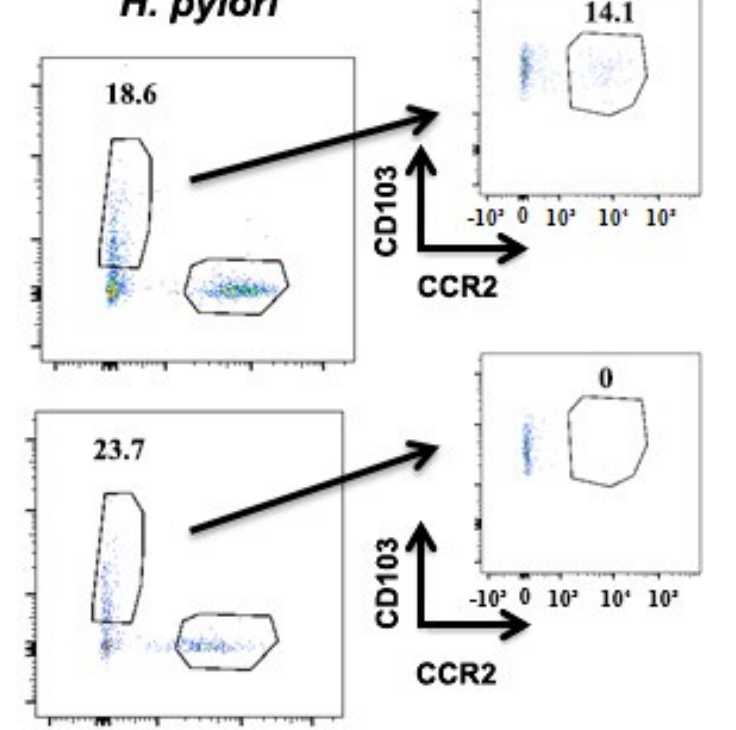

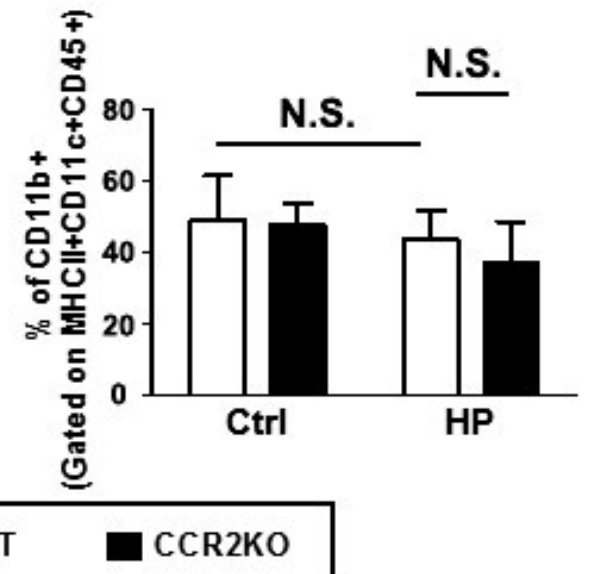

FIGURE 1 Acute H. pylori Infection Induced Upregulation of CCR2 Expression in Mouse Stomach but Deficiency in CCR2 Did not Prevent DC Accumulation in H. pylori-infected Stomach. A, Diagram of the experimental procedures: Stomachs from uninfected or 24-h infected $H$. pylori C57BL/6 mice were removed and $\mathrm{PPCR}$ and FACS analyses were performed to measure the expression of CCR2. B, Total gastric CCR2 relative mRNA expression was increased 24 hours post-H. pylori infection. C, Single-cell prep of stomach from uninfected or $\mathrm{H}$. pylori-infected C57BL/6 mice were analyzed by FACS using fluorochrome-conjugated anti-mouse antibodies (MHCII, CD11c, and CD45 triple-labeled cells were gated and analyzed for CD103 and CD11b expression). CCR2 expression of CD103-labeled cells was also shown (right panels). D, DC and macrophage recruitment to the gastric tissue during $\mathrm{H}$. pylori infection by FACS analysis (gated on CD45-labeled cells). Data are presented as mean \pm SEM, $\mathrm{n}=5 .{ }^{* *} P<.01$ and ${ }^{* * *} P<.001$ compared with uninfected mice 
(A)

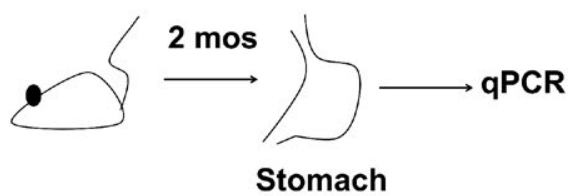

(C)

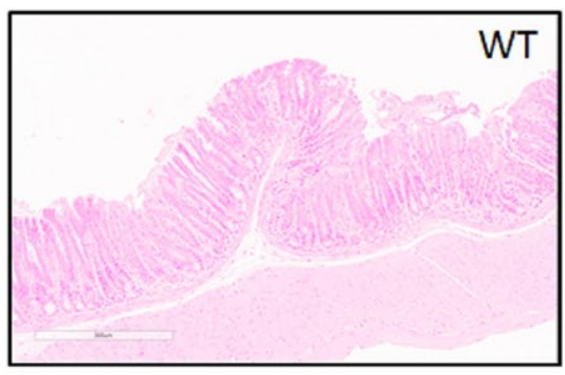

(D)

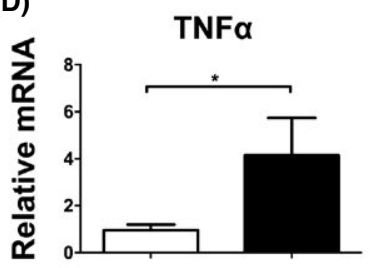

IFN-Y

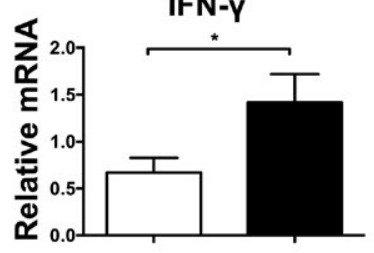

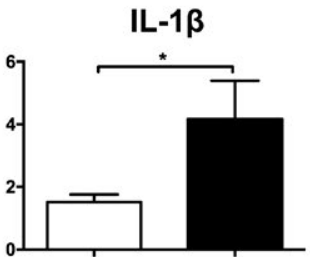

IL-17A

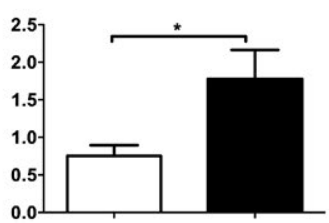

(B)
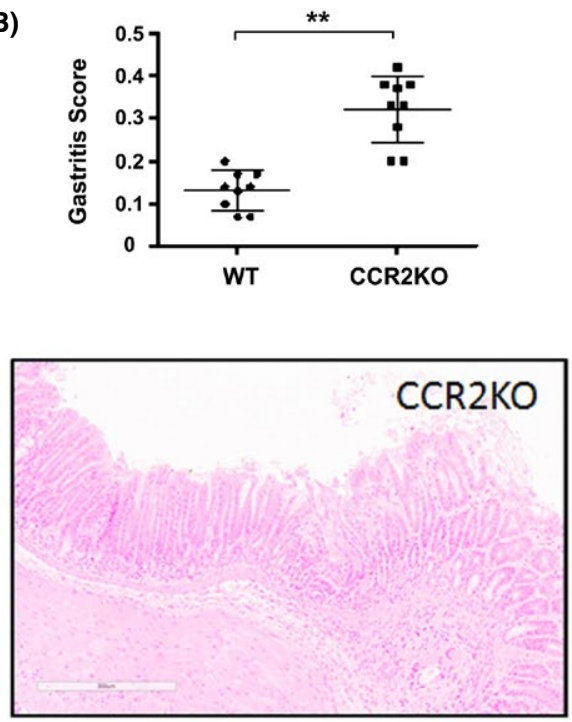

(E)
IL-12p35

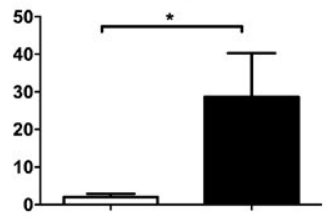

IL-6

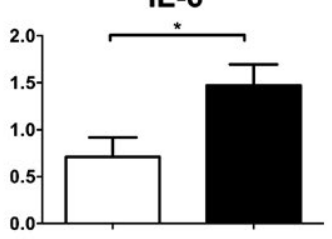

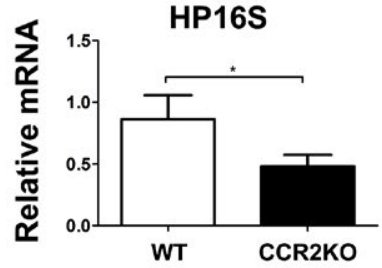

WT

CCR2KO

FIGURE 2 CCR2 Deficiency Increased H. pylori-Induced Gastritis and Decreased H. pylori Colonization. A, Diagram of the experimental procedures: $\mathrm{C} 57 \mathrm{BL} / 6$ mice were orally challenged thrice with $\mathrm{H}$. pylori SS1 and stomachs from Wt mice and CCR2KO mice were removed after eight weeks. B, Gastritis score was determined in a blinded fashion. C, Micrographs of gastric histology. D, mRNA expression of proinflammatory cytokines relative to GAPDH was measured by qPCR. D, H. pylori colonization was determined by quantitative PCR of $H$. pylori $16 \mathrm{~S}$. Results are shown as mean \pm SEM, $n=6-10$ in duplicate. ${ }^{*} P<.05$ and ${ }^{* *} P<0.01$ compared with Wt mice

\subsection{BMDC derived from CCR2KO mice remained immature and failed to induce $H$. pylori-stimulated Treg differentiation in vitro}

Because CCR2 deficiency did not prevent DC recruitment, we hypothesize that CCR2 signaling on DC may impact its function and assessed BMDC function (upregulation of costimulatory molecules, cytokine secretion, and splenic T-cell differentiation) after in vitro H. pylori stimulation. Overnight stimulation of BMDC by $H$. pylori revealed a failure of CCR2KO BMDC to upregulate costimulatory molecules CD80 and CD86 and a lower production of proinflammatory cytokines IL-1 $\beta$ and IL-6 (Figure 4A). Assessment of the function of BMDC in priming $H$. pylori-specific adaptive T-cell responses was performed by coculturing $\mathrm{H}$. pylori-stimulated BMDC with naïve splenocytes for 72 hours in a mixed leukocyte reaction. As shown in Figure 4B,C, CCR2 deficient H. pylori-stimulated BMDC have reduced priming of $H$. pylori-specific adaptive $\mathrm{T}$-cell responses most pronounce in Th1 (IFN- $\gamma$ ) and Treg (Foxp3) responses. The Treg/ Th17 balance was significantly lowered in CCR2KO BMDC compared to Wt BMDC (Figure 4D). To further investigate whether the observed BMDC dysfunction is a result of defective DC differentiation or DC maturation, neutralizing antibodies to MCP-1, a ligand of CCR2, were added to BMDC culture during $H$. pylori stimulation to disrupt CCR2 signaling. MCP-1 depletion interfered with BMDC maturation indicated by lower surface maturation markers CD80 and CD86 as well as a lower IL-6 production of $H$. pylori-stimulated BMDC in anti-MCP-1 treated group vs nontreated group (Fig. S1). These results bring to light the importance of CCR2 signaling in mediating DC maturation and skewing the Treg/Th17 balance toward Treg dominance favoring $H$. pylori tolerance. 
(A)

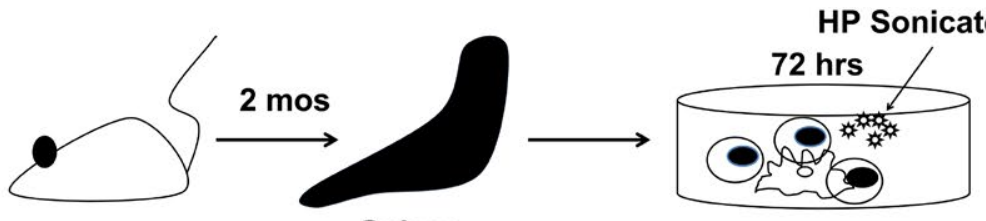

Spleen

(B)

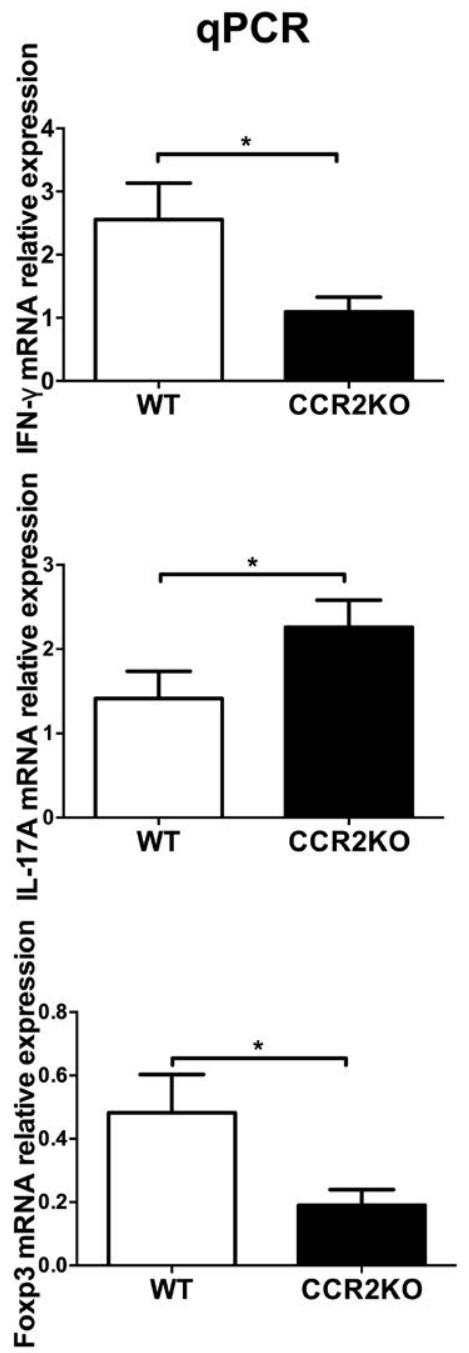

FACS
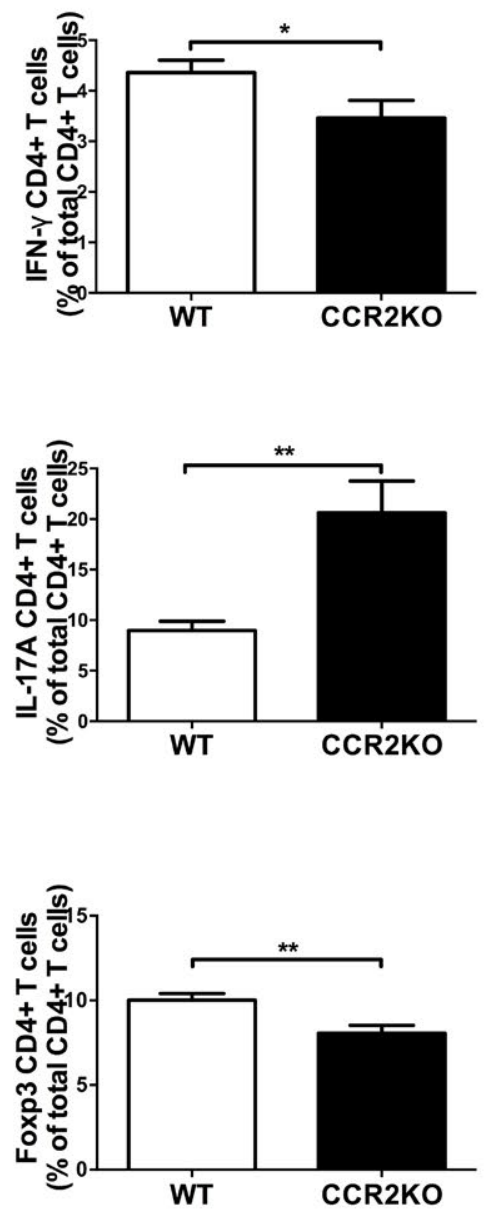

(C)

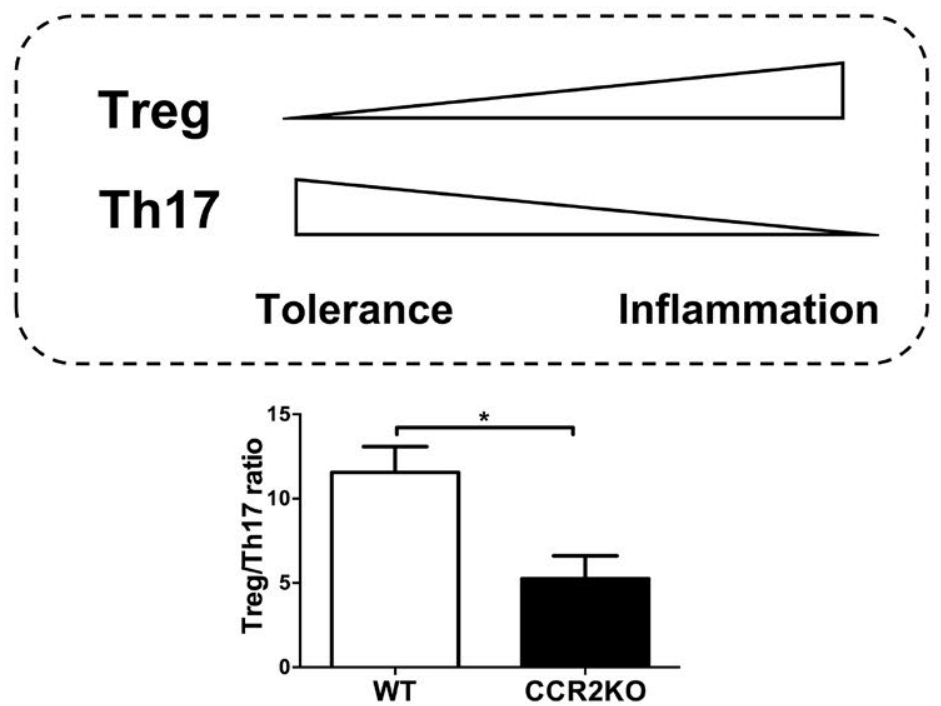

FIGURE 3 CCR2 Deficiency Skews H. pylori-Specific Treg/Th17 Response toward Th17 Immunity. A, Diagram of experimental procedure: Splenocytes from Wt mice or CCR2KO mice after twomonth $\mathrm{H}$. pylori infection were cultured for 72 hours with $H$. pylori sonicate. B, The mRNA expression of Th1 (IFN- $\left.\gamma^{+}\right)$, Th17 $\left(\mathrm{IL}-17 \mathrm{~A}^{+}\right)$, and Treg (Foxp ${ }^{+}$) markers from cocultured splenocytes was determined using $\mathrm{qPCR}$ and FACS analysis showing increased IL-17A and decreased IFN- $\gamma$ and Foxp3 expressions in CCR2KO splenocytes compared to Wt splenocytes. C, The ratio of Treg to Th17 response was calculated using data obtained from FACS (multiplied by 10). Dotted insert illustrates high Treg to Th17 ratio favors $H$. pylori survival and low Treg to Th17 favors H. pylori eradication. Data are expressed as mean \pm SEM, $\mathrm{n}=5$ in duplicate. ${ }^{*} P<.05$ and ${ }^{* *} P<.01$ in comparison with Wt group 
(A)

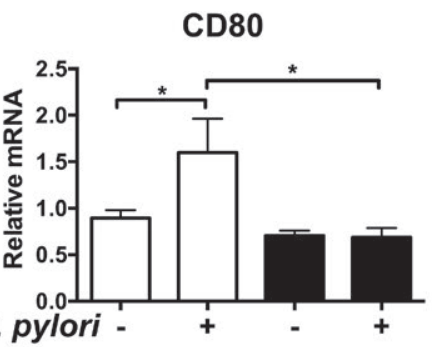

IL-1 $\beta$

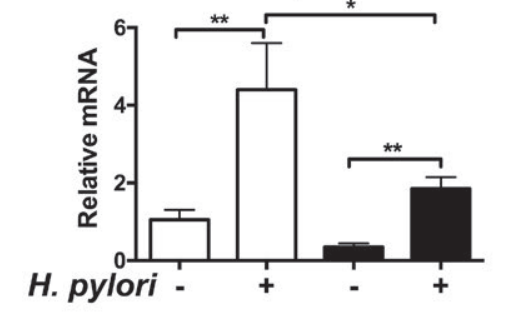

(B)

IFN-Y

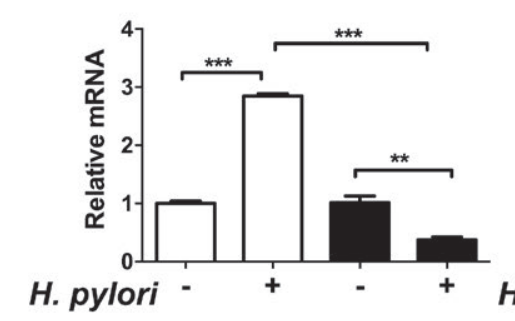

(C)

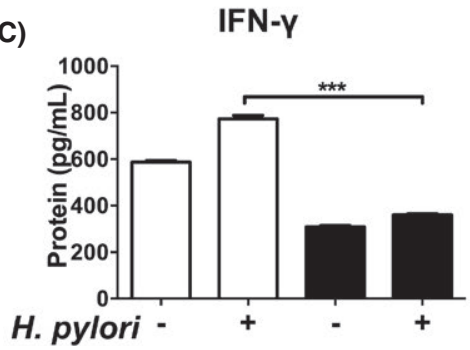

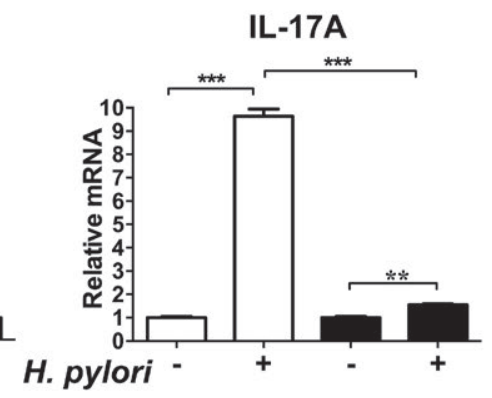
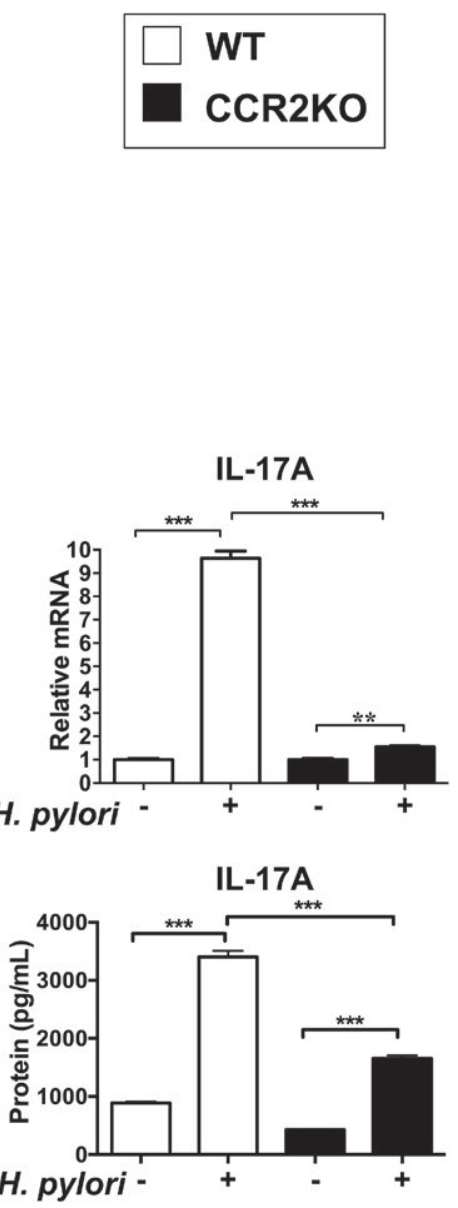

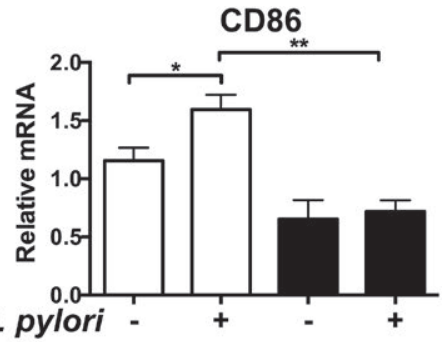

IL-6

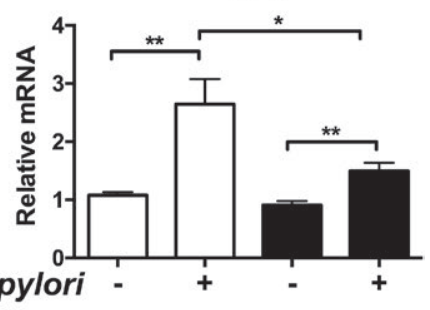

Foxp3

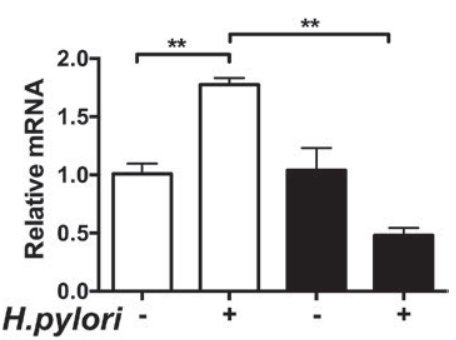

(D)

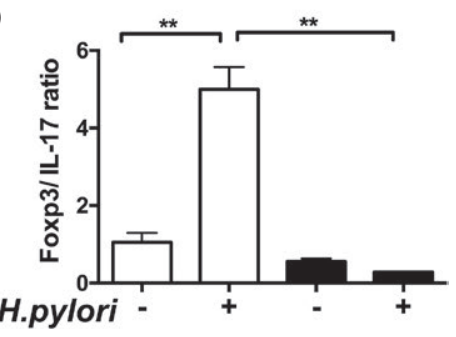

FI GURE 4 BMDC Derived from CCR2KO Mice Remained Immature and Failed to Induce H. pylori-stimulated Treg Differentiation in vitro. BMDCs from Wt and CCR2KO mice $\left(10^{6}\right.$ cells $\left./ \mathrm{mL}\right)$ were stimulated with $\mathrm{H}$. pylori for 18 hours. Cells and Supernatants were collected, and qPCR and ELISA measured mRNA and protein expression. A, BMDC levels of CD80 and CD86 were not increased, and IL-1 $\beta$ production and IL-6 production were diminished in CCR2KO vs Wt mice. BMDCs derived from CCR2KO or Wt mice were stimulated with $H$. pylori and then cocultured with naïve Wt splenocytes for 72 hours. The expression of IFN- $\gamma$, IL-17A, and Foxp3 was measured by qPCR B, or ELISA C. D, BMDC-induced Treg response was lower in CCR2KO vs Wt mice. ${ }^{*} P<.05,{ }^{* *} P<.01$, and ${ }^{* * *} P<.001$ compared to Wt mice. Data are presented as mean \pm SEM of three independent experiments

\section{4 | DISCUSSION}

The current study investigates the role of CCR2 in H. pylori-induced tolerogenic response. We showed that acute $H$. pylori infection was associated with a threefold increase in CCR2 mRNA expression in the gastric mucosa compared to uninfected control mice. CCR2KO mice developed a higher degree of mucosal inflammatory responses and had a lower degree of gastric $H$. pylori colonization with two-month infection compared to infected Wt mice. H. pylori-specific immune response in the CCR2KO spleen was characterized by a significant increase in Th17 response and a decrease in Treg response. We detected recruitment of monocytes in CCR2KO mice and speculated that the decrease in Treg skewing is likely not the result of a lack of dendritic cell trafficking. We then investigated the role of CCR2 signaling on $\mathrm{H}$. pylori-induced DC Treg induction and found that CCR2KO BMDC had lower surface expression of costimulatory molecules (CD80 and CD86) expression and a loss of Treg priming function. These findings indicate that CCR2 signaling in DC supports the induction of Treg and innately maintains mucosal homeostasis which may explain the increased severity of gastritis observed in $\mathrm{H}$. pylori-infected 
CCR2KO mice. Our results indicate that CCR2 plays an essential role in $\mathrm{H}$. pylori-induced tolerance and shed light on a novel mechanism of CCR2-dependent DC Treg induction.

The phenotype of DC plays a major role in the initiation of immune responses in that immature DC are believed to induce tolerance to self-antigens, whereas mature DC promote immunity to foreign and self-antigens. ${ }^{23}$ Fully mature DCs upregulate costimulatory and adhesion molecules (eg, CD40, CD80, and CD86) and MHC class II antigens, and a higher production of cytokines such as IL-12p70, but decrease antigen uptake and processing, ${ }^{24,25}$ which modulates T-cell responses promoting either Th1-cell or cytotoxic T-cell development. Compared to Wt BMDC, we observed decreased surface expression of CCR2KO BMDC maturation markers CD80 and CD86 after H. pylori stimulation, suggesting that CCR2 signaling may be required for DC maturation upon stimulation by bacterial antigens. Moreover, H. pylori-stimulated CCR2KO BMDC are incapable of inducing adaptive T-cell responses including Treg. Similar observations have been reported by Chiu BC et al. that DC-derived CCR2 deficient mice had a lower expression of CD40 and MHCII and a lower level of IL-12 with impaired T-cell responses postpathogen challenge in the lungs. ${ }^{26}$ However, unlike immature DC which induce Treg responses, ${ }^{27}$ CCR2KO DC are unable to produce a robust Treg response possibly contributing to the lower Treg/Th17 balance observed in CCR2KO mice with increased gastritis.

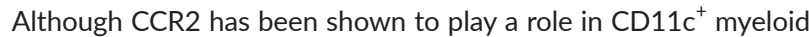
cell recruitment to the mucosal tissue sites in prior studies, ${ }^{12-16}$ it was recently found that CCR2 actively recruits monocytes out of bone marrow compartment and does not affect tissue recruitment. ${ }^{28}$ In our study, we also did not observe a significant difference in DC or monocyte recruitment in $\mathrm{H}$. pylori-infected CCR2KO and Wt mice. Our in vitro studies did reveal a novel role of CCR2 signaling in DC maturation and Treg priming which are necessary for maintenance of homeostasis.

In conclusion, our findings show that $\mathrm{H}$. pylori infection in CCR2KO mice exhibits a lower H. pylori-specific Treg response resulting in increased gastritis and reduced $H$. pylori colonization. We also found that CCR2 signaling is essential for DC maturation and function, which are critical for $\mathrm{H}$. pylori host immune escape. Our study demonstrates a novel mechanism of the pathogenesis of $H$. pylori infection in which CCR2 signaling represents an important target for the modulation of the host response to $H$. pylori.

\section{ACKNOWLEDGEMENTS}

This work was primarily supported by the National Institute of Diabetes and Digestive and Kidney Diseases of the National Institutes of Health under award number R01 DK087708-01 (JYK). Additional funding obtained by XS includes the National Natural Science Foundation, China (81302770), the Postdoctoral Science Foundation, China (2014M551917), and Postdoctoral Innovation Project, Shandong, China (201402029).

\section{COMPETING INTERESTS}

The authors declare that they have no competing interests.

\section{AUTHOR CONTRIBUTIONS}

JYK involved in study concept and design; analysis and interpretation of data; study supervision; and obtained funding; XS involved in drafting of the manuscript; performed experiments; and analysis and interpretation of data; and MZ, ME and GBH assisted with experimental design and data interpretation.

\section{REFERENCES}

1. Perez-Perez GI, Dworkin BM, Chodos JE, Blaser MJ. Campylobacter pylori antibodies in humans. Ann Intern Med. 1988;109:11-17.

2. Suerbaum S, Michetti P. Helicobacter pylori infection. N Engl J Med. 2002;347:1175-1186.

3. Lundgren A, Suri-Payer E, Enarsson K, Svennerholm AM, Lundin BS. Helicobacter pylori-specific CD4+ CD25high regulatory $T$ cells suppress memory $\mathrm{T}$-cell responses to $\mathrm{H}$. pylori in infected individuals. Infect Immun. 2003;71:1755-1762.

4. Raghavan S, Suri-Payer E, Holmgren J. Antigen-specific in vitro suppression of murine Helicobacter pylori-reactive immunopathological $\mathrm{T}$ cells by CD4CD25 regulatory T cells. Scand J Immunol. 2004;60:82-88.

5. Lundgren A, Stromberg E, Sjoling A, et al. Mucosal FOXP3-expressing CD4+ CD25high regulatory $T$ cells in Helicobacter pylori-infected patients. Infect Immun. 2005;73:523-531.

6. Rad R, Brenner L, Bauer S, et al. CD25+/Foxp3+ T cells regulate gastric inflammation and Helicobacter pylori colonization in vivo. Gastroenterology. 2006;131:525-537.

7. Kandulski $A$, Wex $T$, Kuester $D$, et al. Naturally occurring regulatory $T$ cells (CD4+, CD25high, FOXP3+) in the antrum and cardia are associated with higher $\mathrm{H}$. pylori colonization and increased gene expression of TGF-beta1. Helicobacter. 2008;13:295-303.

8. Harris PR, Wright SW, Serrano C, et al. Helicobacter pylori gastritis in children is associated with a regulatory $\mathrm{T}$-cell response. Gastroenterology. 2008;134:491-499.

9. Kao JY, Zhang M, Miller MJ, et al. Helicobacter pylori immune escape is mediated by dendritic cell-induced Treg skewing and Th17 suppression in mice. Gastroenterology. 2010;138:1046-1054.

10. Kao JY, Rathinavelu S, Eaton KA, et al. Helicobacter pylori-secreted factors inhibit dendritic cell IL-12 secretion: a mechanism of ineffective host defense. Am J Physiol Gastrointest Liver Physiol. 2006;291:G73-G81.

11. Fiorina $P$, Jurewicz $M$, Vergani $A$, et al. Phenotypic and functional differences between wild-type and CCR2-/- dendritic cells: implications for islet transplantation. Transplantation. 2008;85:1030-1038.

12. Bruhl $\mathrm{H}$, Cihak J, Schneider MA, et al. Dual role of CCR2 during initiation and progression of collagen-induced arthritis: evidence for regulatory activity of CCR2+ T cells. J Immunol. 2004;172:890-898.

13. Quinones MP, Estrada CA, Kalkonde Y, et al. The complex role of the chemokine receptor CCR2 in collagen-induced arthritis: implications for therapeutic targeting of CCR2 in rheumatoid arthritis. J Mol Med. 2005;83:672-681.

14. Okamoto M, Fuchigami M, Suzuki T, Watanabe N. A novel C-C chemokine receptor 2 antagonist prevents progression of albuminuria and atherosclerosis in mouse models. Biol Pharm Bull. 2012;35:2069-2074.

15. D'Ambrosio D, Panina-Bordignon P, Sinigaglia F. Chemokine receptors in inflammation: an overview. J Immunol Methods. 2003;273:3-13.

16. Charo IF, Ransohoff RM. The many roles of chemokines and chemokine receptors in inflammation. N Engl J Med. 2006;354:610-621.

17. Kurihara T, Warr G, Loy J, Bravo R. Defects in macrophage recruitment and host defense in mice lacking the CCR2 chemokine receptor. J Exp Med. 1997;186:1757-1762.

18. Sato N, Ahuja SK, Quinones M, et al. CC chemokine receptor (CCR)2 is required for langerhans cell migration and localization of $T$ helper cell type 1 (Th1)-inducing dendritic cells. Absence of CCR2 shifts the Leishmania major-resistant phenotype to a susceptible state 
dominated by Th2 cytokines, b cell outgrowth, and sustained neutrophilic inflammation. J Exp Med. 2000;192:205-218.

19. Zhang M, Berndt BE, Eaton KA, Rathinavelu S, Pierzchala A, Kao JY. Helicobacter pylori-pulsed dendritic cells induce $\mathrm{H}$. pylori-specific immunity in mice. Helicobacter. 2008;13:200-208.

20. Eaton KA, Danon SJ, Krakowka S, Weisbrode SE. A reproducible scoring system for quantification of histologic lesions of inflammatory disease in mouse gastric epithelium. Comp Med. 2007;57:57-65.

21. Livak KJ, Schmittgen TD. Analysis of relative gene expression data using real-time quantitative PCR and the 2(-Delta Delta C(T)) Method. Methods. 2001;25:402-408.

22. Dillon S, Agrawal S, Banerjee $\mathrm{K}$, et al. Yeast zymosan, a stimulus for TLR2 and dectin-1, induces regulatory antigen-presenting cells and immunological tolerance. J Clin Invest. 2006;116:916-928.

23. Steinman RM. The dendritic cell system and its role in immunogenicity. Annu Rev Immunol. 1991;9:271-296.

24. Langenkamp A, Messi M, Lanzavecchia A, Sallusto F. Kinetics of dendritic cell activation: impact on priming of $\mathrm{TH} 1, \mathrm{TH} 2$ and nonpolarized T cells. Nat Immunol. 2000;1:311-316.
25. Moser M, Murphy KM. Dendritic cell regulation of TH1-TH2 development. Nat Immunol. 2000;1:199-205.

26. Chiu BC, Freeman CM, Stolberg VR, et al. Impaired lung dendritic cell activation in CCR2 knockout mice. Am J Pathol. 2004;165: 1199-1209.

27. Jonuleit $H$, Schmitt E, Schuler G, Knop J, Enk AH. Induction of interleukin 10-producing, nonproliferating CD4(+) $T$ cells with regulatory properties by repetitive stimulation with allogeneic immature human dendritic cells. J Exp Med. 2000;192: 1213-1222.

28. Serbina NV, Pamer EG. Monocyte emigration from bone marrow during bacterial infection requires signals mediated by chemokine receptor CCR2. Nat Immunol. 2006;7:311-317.

\section{SUPPORTING INFORMATION}

Additional Supporting Information may be found online in the supporting information tab for this article. 